\title{
Propagation and Wireless Channel Modeling Development on Wide-Sense Vehicle-to-X Communications
}

\author{
Wenyi Jiang, Ke Guan, Zhangdui Zhong, Bo Ai, Ruisi He, Binghao Chen, \\ Yuanxuan Li, and Jia You
}

State Key Laboratory of Rail Traffic Control and Safety, Beijing Jiaotong University, Beijing 100044, China

Correspondence should be addressed to Ke Guan; myecone@hotmail.com

Received 22 June 2013; Revised 6 September 2013; Accepted 11 September 2013

Academic Editor: César Briso Rodríguez

Copyright (C) 2013 Wenyi Jiang et al. This is an open access article distributed under the Creative Commons Attribution License, which permits unrestricted use, distribution, and reproduction in any medium, provided the original work is properly cited.

\begin{abstract}
The need for improving the safety and the efficiency of transportation systems has become of extreme importance. In this regard, the concept of vehicle-to-X (V2X) communication has been introduced with the purpose of providing wireless communication technology in vehicular networks. Not like the traditional views, the wide-sense V2X (WSV2X) communications in this paper are defined by including not only vehicle-to-vehicle (V2V) and vehicle-to-infrastructure (V2I) communications but also train-to-X (T2X) communications constituted of train-to-train (T2T) and train-to-infrastructure (T2I) communications. All the information related to the wide-sense V2X channels, such as the standardization, scenarios, characters, and modeling philosophies, is organized and summarized to form the comprehensive understanding of the development of the WSV2X channels.
\end{abstract}

\section{Introduction}

Over the past few years, both the V2X and T2X have gained popularity in their attempts to improve road safety and railway safety, respectively. As shown in Figure 1, in order to form the comprehensive understanding, V2X and T2X are collected together to constitute the complete concept-widesense vehicle-to-X (WSV2X). The idea behind the WSV2X communications is the deployment of wireless communication technology in vehicular and railway networks. In this manner, the vehicles, trains, and infrastructures build up a wireless network enabling them to exchange controlling and traffic information, such as road obstacles, accidents, and so forth, via the wireless communication links.

Following are the situation of the standardization and the application related to WSV2X.

(i) Intelligent transport systems (ITSs) [1] for V2X communications: ITSs are advanced applications which, without embodying intelligence as such, aim to provide innovative services relating to different modes of transport and traffic management and enable various users to be better informed and make safer, more coordinated, and "smarter" use of transport networks. In order to realize the ITSs, Institute of Electrical and
Electronics Engineers (IEEE) and European Telecommunications Standards Institute (ETSI) have selected the same MAC and PHY layers for road traffic safety applications: IEEE 802.11p [2] and ETSI ITS G5 [3]. In the USA, in 1999, the Federal Communications Commission (FCC) allocated $75 \mathrm{MHz}$ of licensed spectrum, from 5.85 to $5.925 \mathrm{GHz}$, as part of the ITS to use for Dedicated Short Range Communications (DSRC) [4]. Hence, IEEE 802.11p, part of the Wireless Access in Vehicular Environments (WAVE) initiative [5], is developed to operate at this $5.9 \mathrm{GHz}$ band, with $75 \mathrm{MHz}$ bandwidth and seven $10 \mathrm{MHz}$ channels. In Europe, "ITS-G5 mode of operation" is defined by ETSI ES 202663 (European profile standard for PHY and MAC layer of $5 \mathrm{GHz}$ ITS), operating at the $5.9 \mathrm{GHz}$ band, with $30 \mathrm{MHz}$ bandwidth and five $10 \mathrm{MHz}$ channels. Systems based on IEEE 802.11p as well as alternative systems are also being developed in the USA, European Union, and some Asian countries.

(ii) For T2X communications, the three main standard systems are communication based train control (CBTC) system [6], global system for mobile communications railway (GSM-R) [7], and Terrestrial 


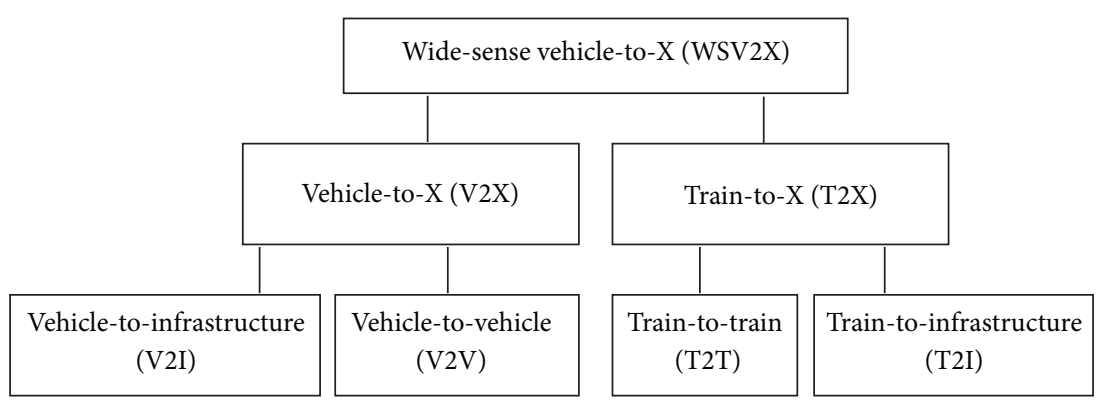

FIGURE 1: Block diagram of the constitution of the concept of wide-sense vehicle-to-X (WSV2X).

Trunked Radio (TETRA, formerly known as TransEuropean Trunked Radio [8]). (i) As one part of European Train Control System (ETCS) [9], CBTC is a railway signalling system that makes use of the telecommunications between the train and track equipment for the traffic management and infrastructure control. By means of the CBTC systems, the exact position of a train is known more accurately than with the traditional signalling systems. This results in a more efficient and safe way to manage the railway traffic. Normally, CBTC works at $2.4 \mathrm{GHz}$. (ii) As a subsystem of European Rail Traffic Management System (ERTMS) [10], GSM-R is an international wireless communications standard for railway communication and applications, used for communication between train and railway regulation control centers. GSM-R is based on GSM and thus works at the $900 \mathrm{MHz}$ band. In EIRENE-MORANE specifications, GSM-R can guarantee performance at speeds up to $500 \mathrm{~km} / \mathrm{h}$, without any communication loss. (iii) As a standard of ETSI, TETRA is a professional mobile radio and two-way transceiver (colloquially known as a walkie talkie) specification. TETRA was specifically designed for use by government agencies, emergency services (police forces, fire departments, and ambulance) for public safety networks, rail transportation staff for train radios, transport services, and the military. Normally, TETRA works at the $400 \mathrm{MHz}$ band.

(iii) So far, there is no official standard for T2T communications, but on the basis of the thought of the ad hoc intervehicle communication, many academic efforts have been made to achieve the direct T2T communications. The most representing system is called Railway Collision Avoidance System (RCAS) [11], studied by Germen Aerospace Center (DLR). Using the global satellite navigation system GALILEO, this system determines and broadcasts information about position, movement vector to other trains around in its coverage for collision detection. When a collision is detected, the train transmits prewarning messages to others to avoid the accident.

(iv) All the standard systems mentioned above are narrowband systems or at least are only implemented at the stage of the narrowband. However, various wideband systems, such as Long Term Evolution for
Railway (LTE-R) $[12,13]$ and LTE for V2X communications, are researched and under industrial consideration. Railway operators do benefit from the evolution in public networks. Fulfilling train control requirements, GSM-R is based on the mature GSM technology. This success is expected to be repeated with LTE/LTE-R [14]. International Union of Railways (UIC) has commenced to generate LTE-R requirements [15]. In [16], the authors discuss several technical challenges for LTE to be used for railroad communications because of fast mobility of train up to $430 \mathrm{~km} / \mathrm{h}$. Since signalling and train control system is related to public safety, very tight reliability requirement needs to be met. Correspondingly, the wideband channel for train communication systems has carried extensive research [17]. Authors in [18] emphasize the feasibility of a smooth evolution from GSM-R to LTE-R. According to the authors, it will not affect the system while the subcarrier interval in LTE is $15 \mathrm{kHz}$, above the $11 \mathrm{kHz}$ recommended. Authors in [19] propose a combination of wireless technologies to provide broadband to the train communication systems. As clarified in [20], the Doppler effect is always a matter of study in this work. Apart from the effect from the academy, many industrial companies have been pushing the standardization of LTE-R, such as the work presented by Nokia Siemense in [14], by Alcatel-Lucent in [21] and by Huawei in [22]. In the near future, the time of the wideband WSV2X communications can be expected to come. For the preparation, the wideband channel characteristics of WSV2X communications are under research by a large number of scholars.

As the basis of WSV2X systems, the propagation and channel characterization are always a fundamental topic with high research interest. In the rest of the paper, all the related information, such as the scenarios, characters, and modeling philosophies of the propagation and wireless channel of WSV2X will be summarized to offer a panorama of the recent progress and the current state of the art. The aim of this paper is to help the designers of communications systems to gain an comprehensive understanding of the pertinent channel characteristics and propagation researchers to assess where the most urgent requirements for further work lie. 


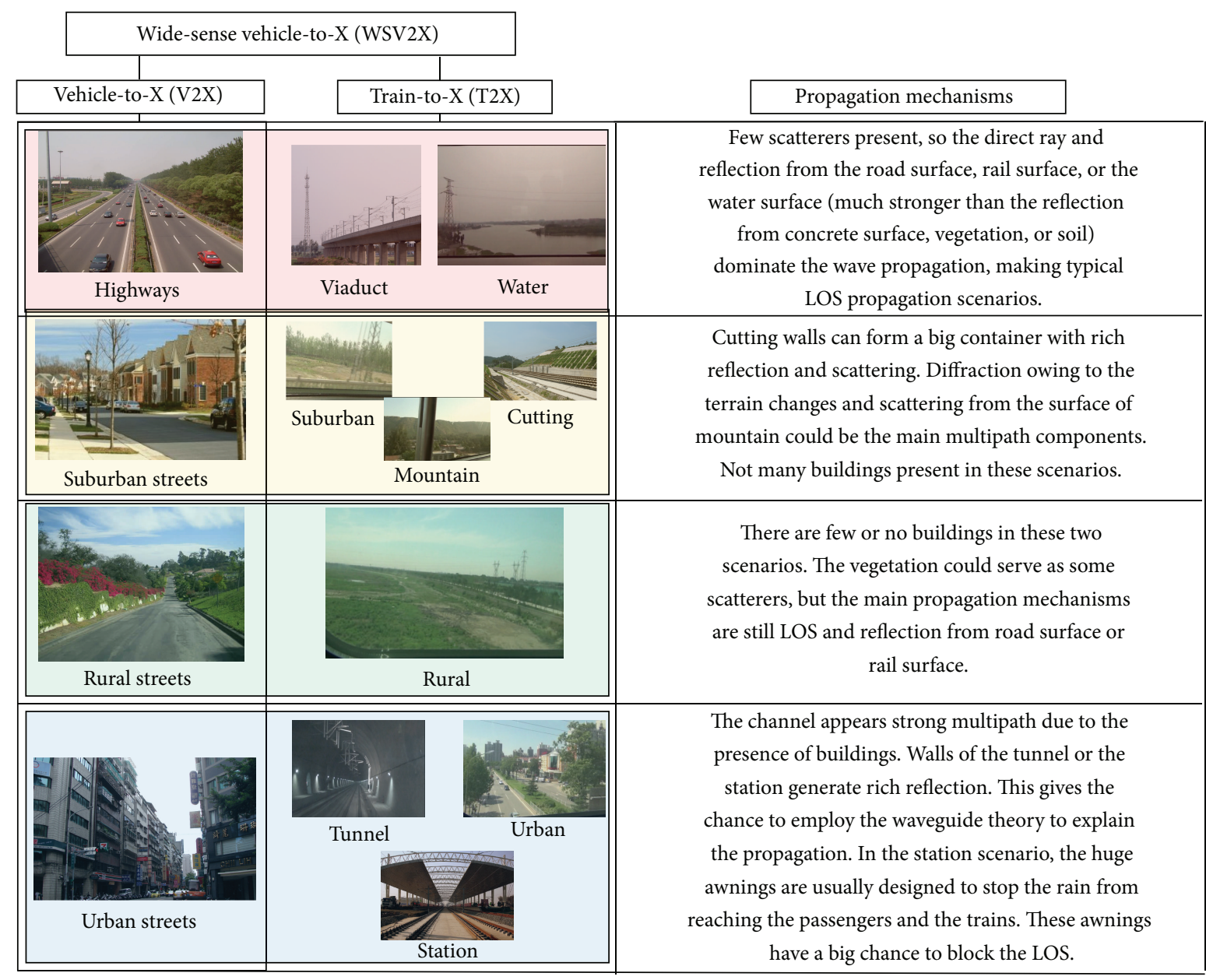

FIgURE 2: Typical scenarios of WSV2X channels. The scenarios in the same light colorful blocks are similar or comparable.

\section{Scenarios of WSV2X Channels}

Channel characteristics of WSV2X channels are dominated by the properties of the scenarios in which the cars, trains, and infrastructures communicate with each other. As shown in Figure 2, there are four main scenarios for the V2X channels (highways, suburban streets, rural streets, and urban streets, defined by [23]) and nine main scenarios for the T2X channels (viaduct, water, suburban, cutting, mountain, rural, tunnel, urban, and station, defined by [24]). The influence of these scenarios on the channel characteristics has been studied by many researchers. For instance, the channel characteristics of V2X in the highways are well summarized by the authors of [23]. The multipath in the rural streets from hills [25] and forests [26] is studied as well. A preliminary table of some narrowband channel characteristics of T2X is given by [24]. For the tunnel scenario, the propagation mechanism changes along with the different distance between transmitter $(\mathrm{Tx})$ and receiver $(\mathrm{Rx})$. Thus, the segmentationbased thought for modeling the propagation inside tunnels has been developed and purchased by many scholars [27-35].

As shown in Figure 2, the scenarios in the blocks with the same color are comparable. They have similarities on the characters of the environments, so the channels are expected to show similar properties. However, up to now the authors cannot find any open publication of doing the comparative study on these channels in the similar scenarios, even though each single scenario has already been well researched independently. Some common senses of the propagation mechanisms in every group of comparable V2X and T2X scenarios are summarized in Figure 2 to give some rough inspirations of the joint analysis of the V2X and T2X scenarios.

In the future, the combination of the similar V2X and $\mathrm{T} 2 \mathrm{X}$ scenarios is expected. In this way, a unified theory of interpreting and modeling the propagation in the WSV2X channels can come true. Thus, the corresponding researchers can use the same methodology and philosophy to characterize the channel, fulfill the network planning, and design the system of WSV2X communication systems.

\section{Comparison of WSV2X Channels with Cellular Communication Channels}

Characteristics of WSV2X channels differ from those of traditional cellular communications channels. These differences 
can be summarized in accordance with the following dimensions.

(i) Heights of Tx and Rx: the relation of heights of Tx and $\mathrm{Rx}$ in T2I channels is very similar to cellular communication (CC) channels- Tx is considerably higher than $\mathrm{Rx}$. However, the $\mathrm{Tx}$ and the $\mathrm{Rx}$ in V2X channel and T2T channel are normally at the same height and in similar environments (peer-topeer communications). This leads to different constitutions of the propagation mechanisms in these channels. (i) LOS: in CC channel and T2I channel, the $\mathrm{Tx}$ is much higher than the Rx. So, compared with V2X and T2T channels, the LOS is relatively easier to be kept in CC channel and T2I channel. (ii) Diffraction: in CC channel and T2I channel, the wave mainly propagates in the vertical plane, so the obstructs are the roof tops of buildings, top of the cutting walls, and terrain changes. Diffraction loss can be effectively estimated by the algorithms based on raster database, such as the classic multiedge models and their improved versions [37]. However, the propagation in V2X and T2T channels mainly happens in the horizontal plane; the diffraction loss owing to street corners and buildings cannot be predicted by raster-based algorithms; vector-based algorithms, such as geometrical theory of diffraction (GTD) and uniform theory of diffraction (UTD), are required. (iii) Distributions of scatterers: normally the area around the Tx in CC channel and T2I channel is free of scatterers, but in V2X and T2T channels, scattering and reflection can come from the scatterers around both the TX and the RX.

(ii) Frequency of communication: compared with the main frequency bands for CC channel (700$2100 \mathrm{MHz}$ ) and T2X channel $(400 \mathrm{MHz}, 900 \mathrm{MHz}$, and $2.4 \mathrm{GHz}$ ), the carrier frequency of $\mathrm{V} 2 \mathrm{X}$ channel which is at $5.9 \mathrm{GHz}$ is very high. Hence, higher signal attenuation occurs in the V2X channel, and specific propagation processes like diffraction are less efficient.

(iii) Distance between $\mathrm{Tx}$ and Rx: in the typical CC and T2I channels, the distance of communication is $1-3 \mathrm{~km}$; however, in V2X and T2T channels, the distance over which communications can take place is normally dozens of meters or hundreds of meters. This makes the V2X and T2T channels more local, and therefore, the scatters far from $\mathrm{Tx}$ and $\mathrm{Rx}$ can be ignored.

(iv) Nonstationarity: in V2X and T2T channels, both Tx and $\mathrm{Rx}$ as well as many scatterers (other vehicles or trains) are dynamic, while in CC channels, only one of the Tx or Rx is moving, and moving scatterers have less relative importance. In T2I channels, the speed of high-speed train can achieve $350 \mathrm{~km} / \mathrm{h}$, which is dramatically faster than the velocity of the user in the CC channel. As a consequence, the channel fluctuations in WSV2X channels are faster, so that the commonly used assumptions on stationarity usually are not valid. The WSV2X channel is the typical nonstationary channel; that is, the channel statistics change within a rather short period of time [23].

\section{WSV2X Channel Characterization}

In the WSV2X channel, all the propagation effects are subsumed into the channel impulse response (CIR), which can be treated as the superposition of the contributions by all multipath components (MPCs). The CIR is time-variant since the channel changes as $\mathrm{Tx}, \mathrm{Rx}$, and scatterers are moving around. A complete description of the channel is thus given by the time variant CIR [38]. In order to facilitate the processing, several metrics are extracted to provide more condensed characterization of the WSV2X channel. Generally speaking, different systems require different metrics to support the channel characteristics. In this paper, we summarize all the typical metrics by classifications of the domains they belong to and the systems they serve.

Figure 3 offers a panorama of metrics for the WSV2X channel classified in accordance with the antenna configuration, bandwidth of the system, and different domains. All the parameters can be assigned to totally six domains:

(i) loss and fading: path loss, shadow fading, and smallscale fading,

(ii) time domain: coherence time and stationary time,

(iii) frequency domain: coherence bandwidth and stationary bandwidth,

(iv) doppler domain: Doppler shift and Doppler spread,

(v) delay domain: RMS delay spread,

(vi) angular domain: angle of arrival (AoA) and angle of departure (AoD).

It can be seen that for the narrow-band single input single output (SISO) systems, the knowledge of path loss, shadow fading, small-scale fading, coherence time, stationary time, coherence bandwidth, stationary bandwidth, and Doppler shift is enough to characterize the channel. But for the wideband SISO systems, Doppler spread and RMS delay spread should be compensated. When the system evolves from SISO to multi-input multioutput (MIMO), the information of the angular domain, such as AoA and AoD is mandatory to 5 characterize the spatial correlations of the subchannels.

Note that the stationary time and the stationary bandwidth are the specific parameters exclusively for the nonstationary channel. Not like the coherence time and the coherence bandwidth which are extracted from the scattering function, the stationary time and the stationary bandwidth measure the amount of Doppler and delay correlation, respectively. They characterize the local scattering function (LSF) variation with respect to time and frequency. In the WSV2X channel, the channel statistics are valid only for a short period of time ("region of stationarity" determined by the stationary time and the stationary bandwidth). For instance, in each region of stationarity, the Doppler spectrum of the first delay tap can be different, because the Doppler shift 


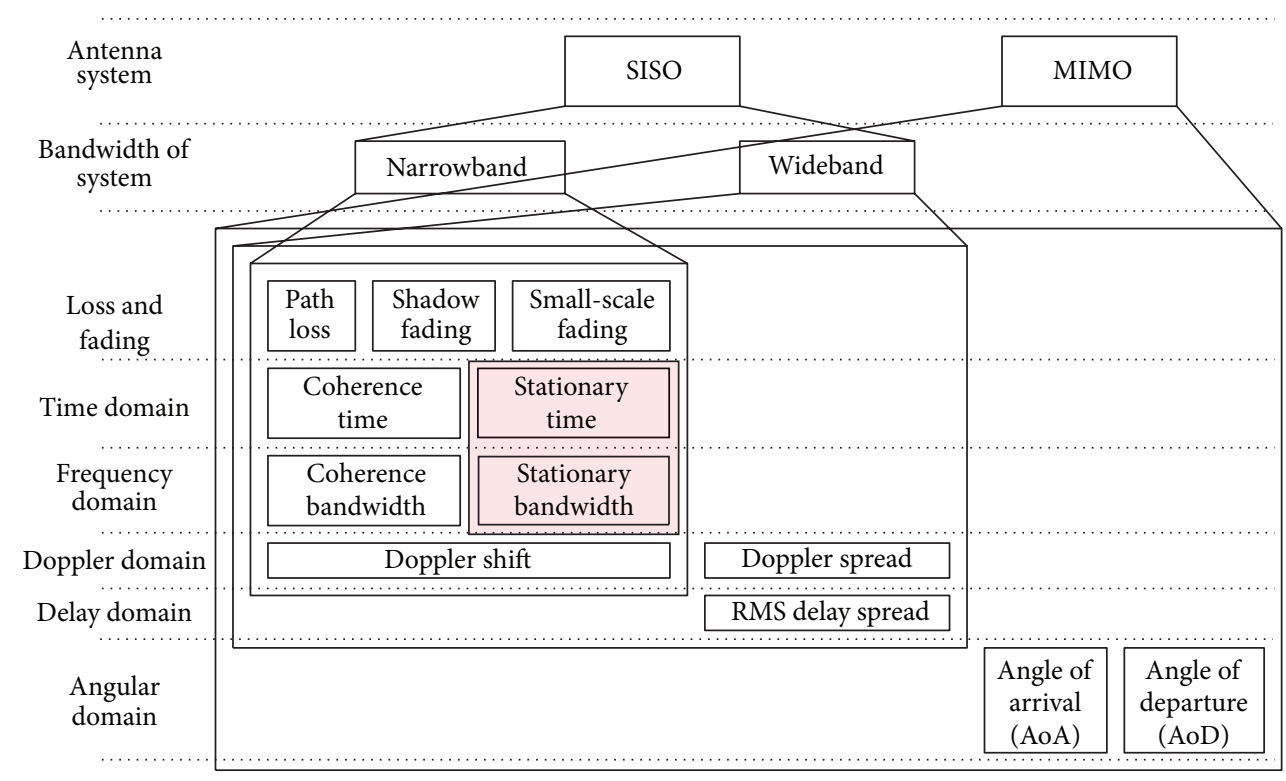

FIgURE 3: Panorama of metrics for WSV2X channel classified in accordance with the antenna configuration and bandwidth of the system and different domains. Stationary time and stationary bandwidth (in the block with light red color) are the two specific parameters in the nonstationary channel.

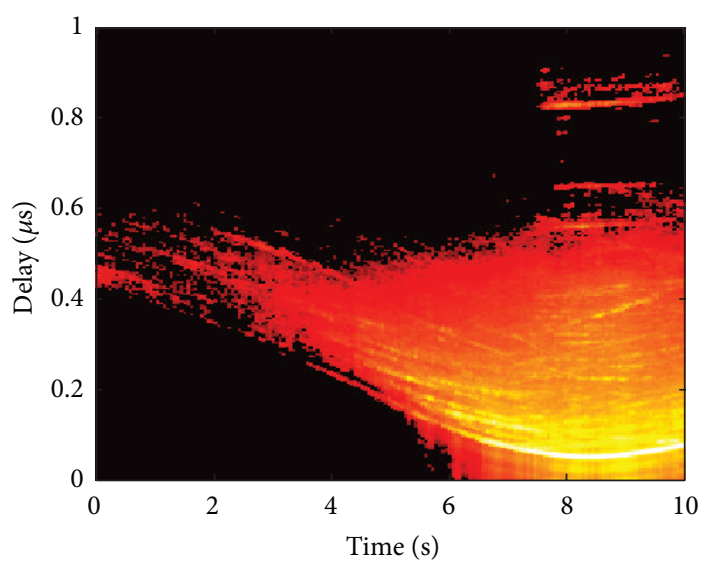

FIGURE 4: Example of the time-varying power delay profile (average squared magnitude of impulse response) in the WSV2X channel. It can be found that the delay of the first taps varies because the Tx and $\mathrm{Rx}$ approach each other firstly and move away from each other later. The LOS tap experiences fading, the gap in the delay domain between strong components (clusters), and the LOS tap changes with time, and there is splitting of clusters over time as well.

of the LOS component can change [23]. Figure 4 (which is the measured results in $[39,40])$ gives an example of the timevarying power delay profile (average squared magnitude of impulse response) in the WSV2X channel. It can be found that the delay of the first taps varies because the Tx and $\mathrm{Rx}$ approach each other firstly and move away from each other later. The LOS tap experiences fading, the gap in the delay domain between strong components (clusters), and the LOS tap changes with time, and there is splitting of clusters over time as well [23].

Up till now, most of the metrics given by Figure 3 in the V2X channel have been well studied by many researchers, such as the metrics given by Table 1 in [23], where the wideband parameters, such as the delay spread and the
Doppler spread, have been extracted from extensive measurements. However, there is no deep insight or comprehensive understanding of these characters for wide-band MIMO T2X communication systems. The authors of [24] offer the narrowband parameters for T2X channels in high-speed railway at $930 \mathrm{MHz}$, but all the wideband parameters are still absent. Some researchers have started to do some efforts, such as the wideband channel characterization of LTE for high-speed railway at $2.35 \mathrm{GHz}$ with the tapped delay line model [41] and the calculation of the stationary interval in high-speed railway scenario [42]. The ergodicity and the reliability of the experimental data and corresponding parameter extractions are still quite limited; more works in different scenarios at various frequencies are expected. 
TABLE 1: Channel characteristics of T2X, V2X, and standard scenarios.

\begin{tabular}{|c|c|c|c|c|c|}
\hline Category & Scenarios & $\begin{array}{l}\text { Path loss } \\
\text { exponent }\end{array}$ & $\begin{array}{l}\text { Standard deviation } \\
\text { of shadowing }[\mathrm{dB}]\end{array}$ & $K$-factor/Nakagami- $m$ & $\begin{array}{c}\text { Delay spread (mean) } \\
{[\mathrm{ns}]}\end{array}$ \\
\hline $\mathrm{T} 2 \mathrm{X}$ & Viaduct & $2-4$ & $2-4$ & $K: 3.8-8.3 \mathrm{~dB}$ & 192 \\
\hline V2X & Highway & $1.8-4$ & $4.0-6.1$ & $K: 3.3-4.3 \mathrm{~dB}$ & $30-340$ \\
\hline $\mathrm{V} 2 \mathrm{X}$ & Rural & $1.79-4$ & - & - & $20-150$ \\
\hline Standard & Suburban & $2.4-4.0$ & $4-8$ & $K: 9 \mathrm{~dB}$ & $59-84$ \\
\hline Standard & $\begin{array}{c}\text { Rural } \\
\text { macrocell }\end{array}$ & $2.2-2.5$ & $4-8$ & $K: 7 \mathrm{~dB}$ & $36.8-42.1$ \\
\hline Standard & $\begin{array}{l}\text { Moving networks } \\
\text { BS-MRS, rural }\end{array}$ & $1.9-2.2$ & 4 & $K: 7 \mathrm{~dB}$ & $40-55$ \\
\hline $\mathrm{T} 2 \mathrm{X}$ & Cutting & $2.5-4$ & $3-5$ & $K: 1.88 \mathrm{~dB}$ & - \\
\hline V2X & Suburban & $2.1-3.9$ & - & $K: 2.1 \mathrm{~dB}$ & $20-150$ \\
\hline Standard & $\begin{array}{l}\text { Indoor to } \\
\text { Outdoor/outdoor to } \\
\text { indoor }\end{array}$ & 2 & 7 & $K: 3.2 \mathrm{~dB}$ & $34-40$ \\
\hline Standard & $\begin{array}{l}\text { Typical urban } \\
\text { macrocell }\end{array}$ & 2.6 & $4-8$ & $K: 7 \mathrm{~dB}$ & 85.4 \\
\hline $\mathrm{T} 2 \mathrm{X}$ & Tunnel & $1.8-3$ & $5-8$ & $m: 0.8-5.4$ & $15-40$ \\
\hline $\mathrm{T} 2 \mathrm{X}$ & Station & $3-5$ & $3-5$ & $K: 0.5-5.4 \mathrm{~dB}$ & - \\
\hline $\mathrm{V} 2 \mathrm{X}$ & Urban & 1.61 & $4.2-6.7$ & $K: 3-6.7 \mathrm{~dB}$ & $3-1100$ \\
\hline Standard & Indoor office/residential & 1.9 & $3-4$ & $K: 7 \mathrm{~dB}$ & $14.2-43$ \\
\hline Standard & $\begin{array}{l}\text { Typical urban } \\
\text { microcell }\end{array}$ & $2.3-4$ & $3-4$ & $K: 9 \mathrm{~dB}$ & $37-74$ \\
\hline Standard & Large indoor/hall & $1.4-3.8$ & $3-4$ & $K: 2 \mathrm{~dB}$ & $23.7-40.8$ \\
\hline
\end{tabular}

Table 1 summarizes some channel parameters of T2X, V2X, and standard scenarios (WINNER) according to the research results of $[23,41,43-47]$, respectively. Not like the traditional way of researching the scenarios of different systems independently, the similar or comparable scenarios of T2X, V2X, and standard scenarios are collected together in Table 1 . There are three preliminary categories.

(i) "Relatively open scenarios": this category is define by the scenarios with a relative wide open space but few scatterers or strong reflectors. The T2X viaduct, V2X highway, V2X rural, standard suburban, standard rural macrocell, and standard moving networks fall into this category. As shown in Table 1, the path loss exponent in this category is slightly larger than 2 , similar to the case of two-ray model. This is because there are few scattering or strong reflections from the surrounding space, but the reflection from the rail surface or the road surface still contributes the power and, therefore, makes the channel close to the two-ray model. Since the LOS can be kept in most of the cases of this category, the Rician $K$-factor is the largest in the three categories. Furthermore, the delay spread is relatively large, because the space is wide.

(ii) "Semiclosed scenarios": this category indicates that the scenario is surrounded by some walls, buildings, or terrain but still has some free or open space. This category is in the middle of the three categories, so the values of the channel characteristics are basically between the "relatively open scenarios" and "relatively closed scenarios."

(iii) "Relatively closed scenarios": this category mainly includes the limited-space and closed scenarios, such as the T2X tunnel, T2X station, V2X urban, standard indoor office/residential, standard typical urban microcell, and standard large indoor/hall. Since these scenarios are mainly in a limited space, the delay spread is relatively small. Moreover, the tunnel walls, station structures, and building walls constitute a relatively closed space, which generates and contains the multipath waves and finally results in a relatively small Rician $K$-factor. Last but not least, most of the scenarios in this category have the path loss exponent smaller than 2; this indicates that certain waveguide effect is established by the surrounding walls of tunnels, stations, and buildings. This makes the path loss even smaller than the free space propagation.

This is the first effort to break the barrier of the V2X, $\mathrm{T} 2 \mathrm{X}$, and standard scenarios. As shown in Table 1, the new classification integrates the scenarios of different systems and redivides the categories according to the physical structure and propagation mechanisms. In the future, the comparative study is expected to gain a deeper insight to the channels in various scenarios and finally form a comprehensive concept of this work. 


\section{Modeling Approach for WSV2X Channel}

There are primarily three types of channel modeling approaches for WSV2X channel: deterministic channel models, stochastic channel models, and geometry-based stochastic models.

\subsection{Deterministic Channel Model for WSV2X Channels.} Deterministic channel models are based on ray-tracing techniques, which model the propagation channel in a specific location using the geographical and morphological information from a database. This kind of modeling approach was pioneered by Wiesbeck [48-50]. Normally, the 3D ray-optical approach covers the direct path, specular reflections, and diffuse scattering. Specular reflections can be calculated by the image method [51] up to the $n$th order, but in practice, only the first and second order reflections are considered in order to limit the computation time. The diffuse scattering is taken into account on surfaces seen by both the Tx and the Rx. Thus, all surfaces of the structures in the scenario, such as buildings, trees, vehicles, and traffic signs, are divided into tiles, and single scattering processes can be determined. For each ray, full-polarimetric antenna patterns are used and channel polarization matrices are computed. The output of the deterministic channel models for each communication link is a time-variant $\operatorname{CIR} h(\tau, t)$, which well characterizes the frequency-selective channel and can be expressed as

$$
h(\tau, t)=\sum_{k=1}^{N(t)} a_{k}(t) \cdot e^{j\left(2 \pi f \tau_{k}(t)+\varphi_{k}(t)\right)} \cdot \delta\left(\tau-\tau_{k}(t)\right),
$$

where the $k$-th multipath component at time $t$ is formulated by an amplitude $a_{k}(t)$, a delay $\tau_{k}(t)$, and an additional phase shift $\varphi_{k}(t)$. As the car or the train is running, the number of multipath components $(N(t))$ is also time variant. Since the amplitude and the phase term for each multipath component can be combined in a complex coefficient: $A_{k}(t)=a_{k}(t)$. $e^{j\left(2 \pi f \tau_{k}(t)+\varphi_{k}(t)\right)},(1)$ can be rewritten as

$$
h(\tau, t)=\sum_{k=1}^{N(t)} A_{k}(t) \cdot \delta\left(\tau-\tau_{k}(t)\right)
$$

By involving the polarization, $A_{k}(t)$ can be expressed by

$$
A_{k}(t)=L_{k}(t) \cdot \vec{e}\left(\theta_{\mathrm{Rx}, k}, \varphi_{\mathrm{Rx}, k}\right)^{H} \cdot P_{k} \cdot \vec{e}\left(\theta_{\mathrm{Tx}, k}, \varphi_{\mathrm{Tx}, k}\right),
$$

where the superscript $H$ denotes the Hermitian transpose. $P_{k}$ denotes the complex channel polarization matrix that includes the reflection or scattering losses of the considered path. $\vec{e}\left(\theta_{\mathrm{Tx}, k}, \varphi_{\mathrm{Tx}, k}\right)$ and $\vec{e}\left(\theta_{\mathrm{Rx}, k}, \varphi_{\mathrm{Rx}, k}\right)$ are the complex polarization vectors for the $\mathrm{Tx}$ and the $\mathrm{Rx}$, respectively, that cover the gain and the polarization of the antennas at the angles of departure $\left(\theta_{\mathrm{Tx}, k}, \varphi_{\mathrm{Tx}, k}\right)$ and the angles of arrival $\left(\theta_{\mathrm{Rx}, k}, \varphi_{\mathrm{Rx}, k}\right)$ for the $k$-th multipath ray. $L_{k}(t)$ includes the propagation loss and the phase shift based on the delay $\tau_{k}(t)$ of the considered path. For the direct ray, $P_{k}$ is the identity matrix, whereas for reflected and scattered rays of the order $n, P_{k}$ is derived by [52] using

$$
P_{k}=R\left(\varphi_{n-m, k}\right) \cdot \prod_{m=0}^{n-2}\left(R_{n-m, k} \cdot R\left(\varphi_{n-m, k}\right)\right) \cdot R_{1, k} \cdot R\left(\varphi_{\mathrm{Tx}, k}\right),
$$

where $R(\varphi)$ is the rotation matrix that can be expressed as

$$
R(\varphi)=\left(\begin{array}{cc}
\cos \varphi & \sin \varphi \\
-\sin \varphi & \cos \varphi
\end{array}\right)
$$

$\varphi_{\mathrm{Tx}, k}$ denotes the angle between the normal vector of the incidence plane and the field strength component $E_{\theta}$ at the first reflection or scattering point, and $\varphi_{\mathrm{Rx}, k}$ denotes the angle between the scattering or reflecting plane and $E_{\theta}$ at the last reflection or scattering point for the $k$-th multipath component, respectively. For the case of the multiple reflections or scattering, the term $\varphi_{i, k}$ denotes the angle between $E_{\theta}$ and the normal vector of the incidence plane at the $i$ th reflection or scattering point for the $k$-th multipath ray. $R_{i, k}$ denotes the reflection or scattering matrix which is given by

$$
R_{i, k}=\left(\begin{array}{cc}
r_{\perp_{i, k}} & \zeta_{1_{i, k}} \\
\zeta_{2_{i, k}} & r_{\|_{i, k}}
\end{array}\right)
$$

where the elements $r_{\perp_{i, k}}$ and $r_{\|_{i, k}}$ are the reflection or scattering coefficients of the surface belonging to the $i$ th reflection or scattering point of the $k$-th multipath component for perpendicular and parallel components of the electric field, respectively. For reflection processes, these elements are determined based on the Fresnel equations; for scattering processes, these values depend on the radar cross-section (RCS) of the object. $\zeta_{1_{i, k}}$ and $\zeta_{2_{i, k}}$ are cross-polarization coupling coefficients, which are normally set to zero. More detailed information about the deterministic modeling approach can be found in $[53,54]$. Figures 5(a) and 5(b) offer an example of a raytracing map of the realistic high-speed railway scenario, the partial enlarged drawing for direct, reflected, and scattering paths in one snapshot. Figure 5(c) gives a ray-tracing map of a typical urban V2V channel.

5.2. Stochastic Channel Models. The stochastic channel model does not determine the impulse response in a specific location. Rather, it models the propagation characteristics by assuming stochastic distributions of scatterers. Basically, the stochastic channel models can be divided into two types: narrowband stochastic channel models and wideband stochastic channel models.

Narrowband stochastic models do not concern the frequency selectivity of the channel but rather focus on characterization of the fading statistics as well as the Doppler spectrum. Not like the Jakes spectrum in the CC channel with a "bathtub" shape, the Doppler spectrum in V2V channels is more smoother $[55,56]$. More efforts and interesting results are given in $[57,58]$.

Wideband stochastic channel models create the statistics of the received power with a certain delay, Doppler shift, and 


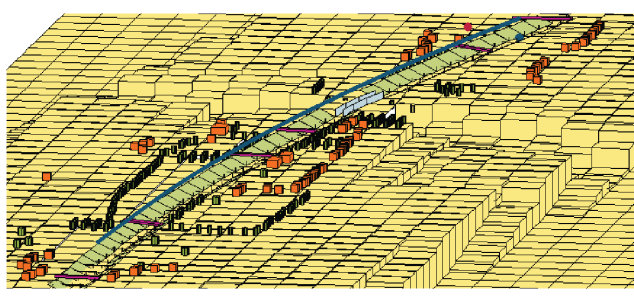

(a)

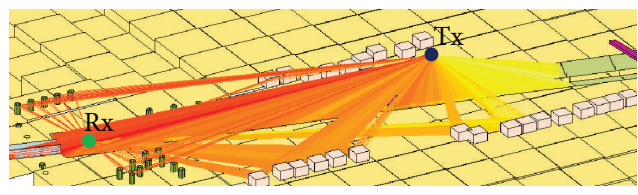

(b)

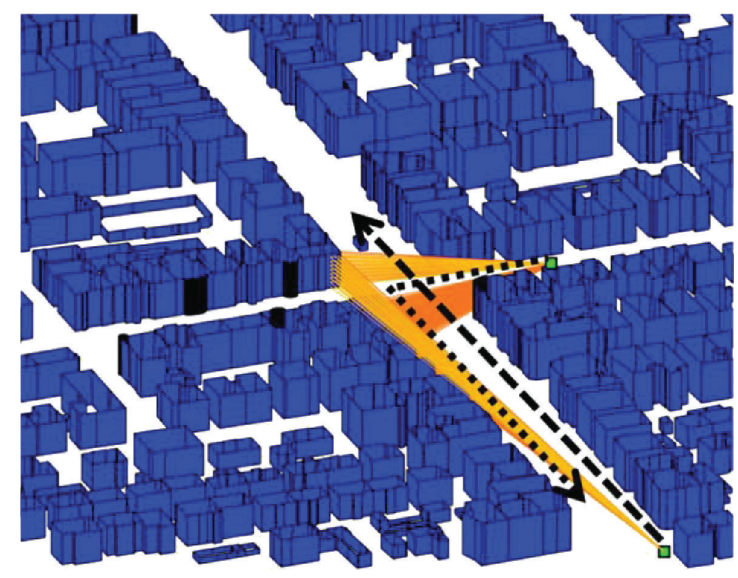

(c)

FIGURE 5: Example of (a) a ray-tracing map of the realistic high-speed railway scenario; (b) partial enlarged drawing for direct, reflected, and scattering paths in one snapshot; (c) and a ray-tracing map of a typical urban V2V channel.

angle of arrival. Based on the wide-sense stationary uncorrelated scattering (WSSUS) assumption [59], the tapped delay line model is developed and widespread used for cellular system simulations. This approach describes the CIR by means of a finite impulse response filter with a number of discrete taps, each of which is fading according to a prescribed probability density function and Doppler spectrum. In particular, the IEEE $802.11 \mathrm{p}$ channel models employ the 6-tap and 12-tap models developed by Ingram and coworkers $[60,61]$ in different types of environments. Since each tap contains multiple paths and each path can have a different type of Doppler spectrum, almost arbitrary Doppler spectra can be synthesized for each tap. Due to the low complexity of the implementation, tapped delay line models are widely used, even though they may suffer from less accurate representation of the nonstationarity in WSV2X channels.

5.3. Geometry-Based Stochastic Models. Geometry-based stochastic modeling (GSCM) is similar to the stochastic modeling, but it uses a simplified ray tracing along with random scatterers. The GSCM is widely used in MIMO channel modeling [38]. Generally, the GSCM models can be divided into the regular-shaped GSCMs (RS-GSCMs) and irregular shaped GSCMs (IS-GSCMs) [62]. It depends on whether the effective scatterers distribute on a regular shape (one-ring, two-ring, and ellipses) or an irregular shape determined by the environment. RS-GSCMs are mathematically tractable and used for theoretical analysis. However, the IS-GSCM can easily handle the nonstationarity of WSV2X channel. Both the RS-GSCM and IS-GSCM models have been researched in the V2X channel and the T2I channel. Figure 6 gives sketches of the geometry-based stochastic model of T2I channel [36] in the cutting scenario and V2V channel [26] with scatterers in realistic positions.

5.4. Summary and Selecting a Suitable Modeling Approach. Each of the modeling methods offered above has specific advantages and disadvantages. (i) Deterministic channel models offer the most accurate simulation of the realistic channel model including the nonstationarity of the channel naturally. However, they require highly accurate topographical databases and are numerically intensive to process. Moreover, the high resolution digital elevation model (DEM) for the ray-tracing method is very expensive, and this limits the application of the deterministic models.

(ii) Stochastic channel models, particularly the tapped delay line models, can be parameterized in a relatively flexible way to describe channels in different scenarios. However, the implementations of the tapped delay line models, especially in the IEEE 802.11 p channel models, do not consider the nonstationarity of the channel. This is the main drawback of the application of such kind of modeling method to the WSV2X channel and, therefore, more and more researchers, such as the authors in [63], are making efforts to introduce the nonstationarity to the stochastic channel models.

(iii) Geometry-based stochastic models can reflect the realistic behavior of the WSV2X channel and implicitly describe the nonstationarity of the channel. They require more computation time than stochastic channel models to realize a CIR but normally are still much easier than the deterministic models.

Hence, it is hard to say which modeling method is the most outstanding. The choice always strongly depends on the concrete requirements of the application and the development of each method. For instance, the stochastic modeling methods can be a very simple and promising choice to realize the channel if the channel does not change fast or the nonstationarity can be successfully involved in the implementation. The deterministic modeling method can be more proper if the network planning or the system level simulation has a very high accurate requirement, and more effective acceleration techniques, such as the parallel processing and 


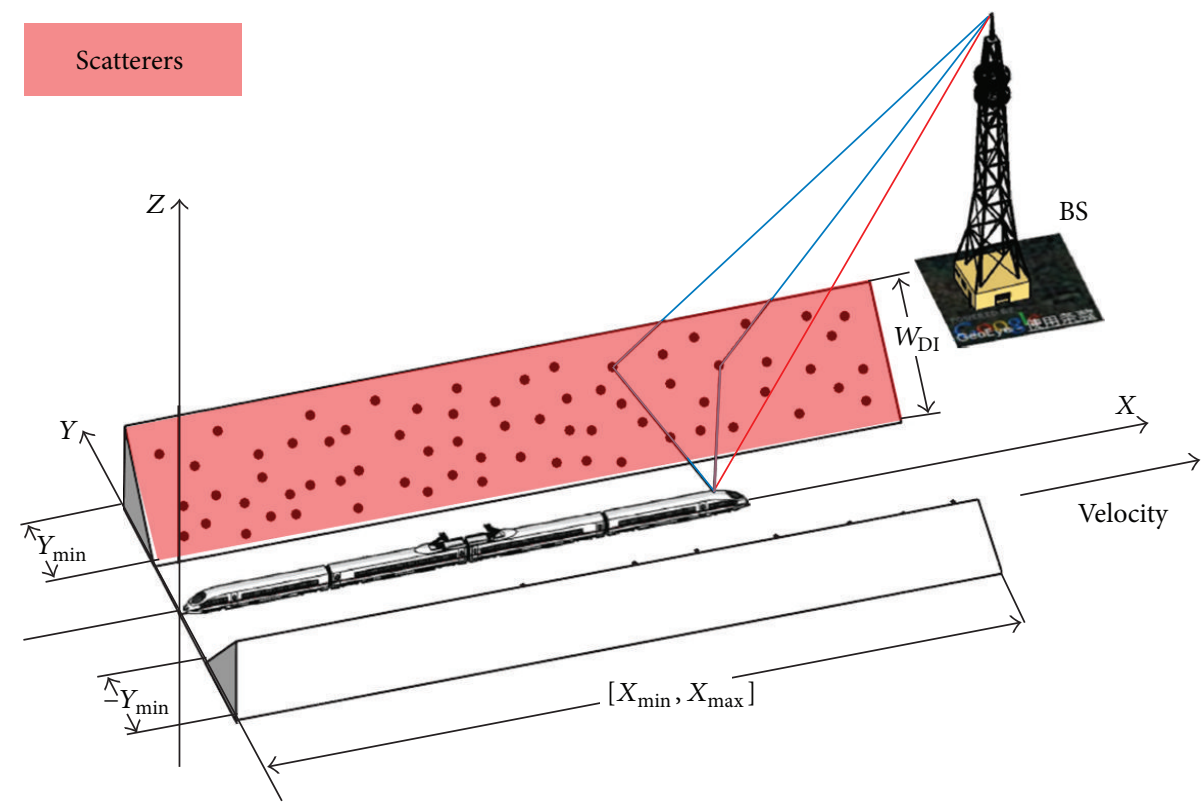

(a)

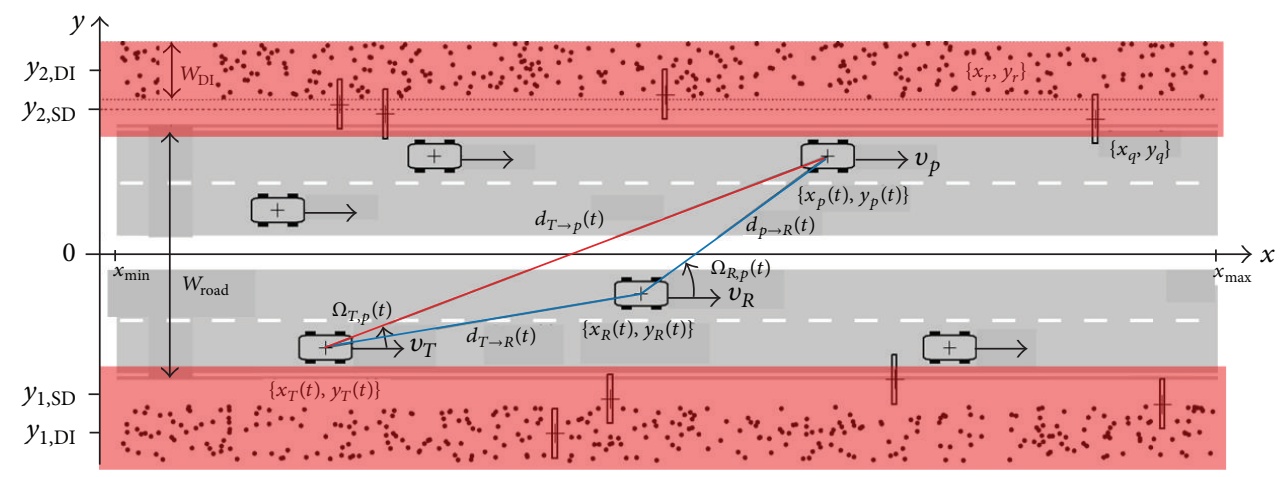

(b)

FIGURE 6: (a) Sketch of the geometry-based stochastic model of T2I channels in the cutting scenario with scatterers in realistic positions [36]. (b) Sketch of the geometry-based stochastic model of V2V channels [26].

backface culling [64], are implemented. According to the current level, the geometry-based stochastic model can be treated as a middle course of the other two kinds of modeling methods in the simulation, but it cannot be used for the network planning or the site-specific simulation.

\section{Conclusion}

Traditional concepts of $\mathrm{V} 2 \mathrm{X}$ and $\mathrm{T} 2 \mathrm{X}$ are summarized together to constitute a more general concept-wide-sense V2X (WSV2X). This paper offers an overview of the development of the WSV2X channels, such as the standardization, scenarios, characters, and modeling approaches. This paper integrates the common senses of V2X, T2X, and standard scenarios and forms a new panorama of the scenarios classified by the similar physical characters and propagation mechanisms. This effort is made to give some rough inspirations of the joint research of the V2X and T2X scenarios. Following this thought, it is expected to interpret and model the WSV2X channels in a uniform way. As a result, the channel characterization, network planning, and system design of WSV2X communication systems can be realized by using the same methodology and philosophy. However, besides these results, the answers of more open questions are still missing. For instance, the developments of the V2X and T2X channels are not balanced, how can we compensate the corresponding research in $\mathrm{T} 2 \mathrm{X}$ channels to fulfill a panorama of theWSV2X channel? How can we describe the nonstationarity of the WSV2X channel in an easy way? How can we break the limitations of each modeling approaches or develop some hybrid models integrating the advantages of various modeling methods? So many open questions, combined with the increasing importance of WSV2X communications, will surely make the WSV2X channels an exciting and promising research field in the next years.

\section{Acknowledgments}

The authors express their thanks to the support from the NNSF of China under Grant U1334202; NNSF of China 
under Grant 61222105; Beijing Municipal NSF under Grant 4112048; the Key Grant Project of Chinese Ministry of Education (no. 313006); Project of State Key Lab under Grants RCS2012ZT013, RCS2011K008, and RCS2011ZZ008; and Key Project for Railway Ministry of China under Grant 2012X008A.

\section{References}

[1] T. Vaa, M. Penttinen, and I. Spyropoulou, "Intelligent transport systems and effects on road traffic accidents: state of the art," IET Intelligent Transport Systems, vol. 1, no. 2, pp. 81-88, 2007.

[2] IEEE Std. 802. 11-2007, "Part 11: weireless lAN medium access control (MAC) and physical layer (PHY) specifications," IEEE Std. 802. 11, 2007.

[3] ETSI ES 202 663, "Intelligent transport systems, european profile standard on the physical and medium access layer of 5 GHz ITS," Draft Version 0. 0. 6, October 2009.

[4] U. S. Federal Communications Commission, "Report and order," Tech. Rep. FCC 03-324, 2003.

[5] IEEE Std 1609. 4, "IEEE standard for wireless access in vehicular environments (WAVE)-multi-channel operation," February 2011.

[6] "IEEE standard for communications-based train control (CBTC) performance and functional requirements," December 1999.

[7] http://www.uic.asso.fr.

[8] ETSI ETR 300-3 (2000-02), “Terrestrial trunked radio (TETRA), voice plus data $(\mathrm{V}+\mathrm{D})$, designers' guide, part 3: Direct Mode Operation (DMO)," 1st edition, 2000.

[9] H. Zhou and B. Luo, "Design and budget analysis of RF receiver of $5.8 \mathrm{GHz}$ ETC reader," in Proceedings of the International Conference on Communication Technology (ICCT '10), pp. 354357, Nanjing, China, November 2010.

[10] ERTMS/ETCS-Class 1, FFFIS for Eurobalise. Subset-026, Issue 2. 4. 1. September 2007.

[11] C. R. García, A. Lehner, T. Strang, and K. Frank, "Channel model for train to train communication using the $400 \mathrm{MHz}$ band," in Proceedings of the 2008 IEEE 67th Vehicular Technology Conference, pp. 3082-3086, Singapore, May 2008.

[12] 1st Year Research Report on Railroad Wireless Communication System, Korea Railroad Research Institute, Seoul, Republic of Korea, 2011.

[13] K. Guan, Z. Zhong, and B. Ai, "Assessment of LTE-R using high speed railway channel model," in Proceedings of the 3rd International Conference on Commnunications and Mobile Coputing, pp. 461-464, Qingdao, China, 2011.

[14] P. Tiberg, GSM-Railway, Yesterday, Today and Tomorrow, Nokia Siemens Networks, Banebranchen, Copenhagen, Denmark, 2009.

[15] http://www.uic.org.

[16] R. Y. Kim, J. S. Kwak, and H. C. Hwang, "Technical challenges of railroad communications using long term evolution," in Proceedings of the IEEE International Conference on ICT Convergence (ICTC '12), pp. 1-2, Jeju, Repablic of Korea, 2012.

[17] M. Aguado, I. Liedo Samper, M. Berbineau, and E. Jacob, “4G communication technologies for train to ground communication services: LTE versus WIMAX, a simulation study," in Proceedings of the 9th World Congress on Railway Research, pp. 1-10, Lille, France, 2011.
[18] T. Gao and B. Sun, "A high-speed railway mobile communication system based on LTE," in Proceedings of the International Conference on Electronics and Information Engineering (ICEIE '10), pp. 414-417, Kyoto, Japan, August 2010.

[19] J. Zhang, Z. Tan, Z. Zhong, and Y. Kong, "A multi-mode multiband and multi-system-based access architecture for highspeed railways," in Proceedings of the IEEE 72nd Vehicular Technology Conference Fall (VTC '10), pp. 1-5, Ottawa, Canada, September 2010.

[20] UIC, GSM-R Procurement Guide, Union Internationale des Chemins de Fer, Paris, France, 2007.

[21] O. Andre, LTE and Its Applications in Railways, Networks and the New Economy, Alcatel-Lucent, Cambridge, UK, 2010.

[22] "Huawei eyes European market," September 2011, http://www .railwaygazette.com/news/business/single-view/view/huaweieyes-european-market.html.

[23] A. F. Molisch, F. Tufvesson, J. Karedal, and C. F. Mecklenbräuker, "A survey on vehicle-to-vehicle propagation channels," IEEE Wireless Communications, vol. 16, no. 6, pp. 12-22, 2009.

[24] B. Ai, R. He, Z. Zhong et al., "Radio wave propagation scene partitioning for high-speed rails," International Journal on Antennas and Propagation, vol. 2012, Article ID 815332, 7 pages, 2012.

[25] L. Cheng, B. E. Henty, D. D. Stancil, F. Bai, and P. Mudalige, "Mobile vehicle-to-vehicle narrow-band channel measurement and characterization of the $5.9 \mathrm{GHz}$ dedicated short range communication (DSRC) frequency band," IEEE Journal on Selected Areas in Communications, vol. 25, no. 8, pp. 1501-1516, 2007.

[26] J. Karedal, F. Tufvesson, N. Czink et al., "A geometry-based stochastic MIMO model for vehicle-to-vehicle communications," IEEE Transactions on Wireless Communications, vol. 8, no. 7, pp. 3646-3657, 2009.

[27] K. Guan, Z. Zhong, B. Ai, and C. Briso, "Propagation mechanism analysis before the break point inside tunnels," in Proceedings of the IEEE 74th Vehicular Technology Conference, pp. 1-5, San Francisco, Calif, USA, 2011.

[28] K. Guan, Z. Zhong, B. Ai, and C. Briso-Rodríguez, "Propagation mechanism modelling in the near region of circular tunnels," IET Microwaves, Antennas and Propagation, vol. 6, no. 3, pp. 355-360, 2012.

[29] K. Guan, Z. Zhong, B. Ai, and C. Briso-Rodríguez, "Modeling of the division point of different propagation mechanisms in the near-region within arched tunnels," Wireless Personal Communications, vol. 68, no. 3, pp. 489-505, 2013.

[30] K. Guan, Z. Zhong, B. Ai, R. He, Y. Li, and C. Briso, "Propagation mechanism modeling in the near-region of arbitrary cross-sectional tunnels," International Journal of Antennas and Propagation, vol. 2012, Article ID 183145, 11 pages, 2012.

[31] K. Guan, Z. Zhong, B. Ai, and C. Briso-Rodríguez, "Research of propagation characteristics of break point; Near zone and far zone under operational subway condition," in Proceedings of the 6th International Wireless Communications and Mobile Computing Conference (IWCMC '10), pp. 114-118, Caen, France, July 2010.

[32] K. Guan, Z. Zhong, B. Ai, and C. Briso-Rodríguez, "Measurement and modeling of subway near shadowing phenomenon," in Proceedings of the 5th International ICST Conference on Communications and Networking in China (ChinaCom '10), pp. 1-5, Beijing, China, August 2010. 
[33] K. Guan, Z. Zhong, B. Ai, and C. Briso, "Novel hybrid propagation model inside tunnels," in Proceedings of the IEEE 75th Vehicular Technology Conference, pp. 1-5, Yokohama, Japan, 2012.

[34] K. Guan, Z. Zhong, B. Ai et al., "Complete propagation model structure inside tunnels," Progress in Electromagnetics Research, vol. 141, pp. 711-726, 2013.

[35] K. Guan, Z. Zhong, B. Ai, R. He, and C. Briso-Rodriguez, "Fivezone propagation model for large-size vehicles inside tunnels," Progress in Electromagnetics Research, vol. 138, pp. 389-405, 2013.

[36] B. Chen and Z. Zhong, "Geometry-based stochastic modeling for MIMO channel in high-speed mobile scenario," International Journal of Antennas and Propagation, vol. 2012, Article ID 184682, 6 pages, 2012.

[37] K. Guan, Z. D. Zhong, B. Ai, and T. Kuerner, "Semi-deterministic propagation modeling for high-speed railway, IEEE Antennas and Wireless Propagation Letters, vol. 12, pp. 789-792, 2013.

[38] P. Almers, E. Bonek, A. Burr et al., "Survey of channel and radio propagation models for wireless MIMO systems," Eurasip Journal on Wireless Communications and Networking, vol. 2007, Article ID 19070, 2007.

[39] T. Abbas, J. Karedal, F. Tufvesson, A. Paier, L. Bernadó, and A. F. Molisch, "Directional analysis of vehicle-to-vehicle propagation channels," in Proceedings of the IEEE 73rd Vehicular Technology Conference (VTC '11), pp. 1-5, Budapest, Hungry, May 2011.

[40] J. Nuckelt, T. Abbas, F. Tufvesson, C. Mecklenbräuker, L. Bernadó, and T. Kürner, "Comparison of ray tracing and channel-sounder measurements for vehicular communications," in Proceedings of the IEEE 77th Vehicular Technology Conference (VTC '13), pp. 1-5, Dresden, Germany, June 2013.

[41] L. Liu, C. Tao, J. Qiu et al., "Position-based modeling for wireless channel on high-speed railway under a viaduct at $2.35 \mathrm{GHz}$," IEEE Journal on Selected Areas in Communications, vol. 30, no. 4, pp. 834-845, 2012.

[42] B. Chen, Z. Zhong, and B. Ai, "Stationarity intervals of timevariant channel in high speed railway scenario," China Communications, vol. 9, pp. 64-70, 2012.

[43] H. Wei, Z. Zhong, K. Guan, and B. Ai, "Path loss models in viaduct and plain scenarios of the high-speed railway," in Proceedings of the 5th International ICST Conference on Communications and Networking in China (ChinaCom '10), pp. 1-5, Beijing, China, August 2010.

[44] K. Guan, Z. Zhong, J. I. Alonso, and C. Briso-Rodríguez, "Measurement of distributed antenna systems at $2.4 \mathrm{GHz}$ in a realistic subway tunnel environment," IEEE Transactions on Vehicular Technology, vol. 61, no. 2, pp. 834-837, 2012.

[45] Y. Guo, J. Zhang, C. Tao, L. I. U. Liu, and L. Tian, "Propagation characteristics of wideband high-speed railway channel in viaduct scenario at 2. $35 \mathrm{GHz}$," Journal of Modern Transportation, vol. 20, no. 4, pp. 206-212, 2012.

[46] G. Acosta-Marum and M. A. Ingram, "Six time- and frequencyselective empirical channel models for vehicular wireless LANs," IEEE Vehicular Technology Magazine, vol. 2, no. 4, pp. 4-11, 2007.

[47] IST WINNER II D1. 1. 2, “WINNER II channel models," http:// www.ist-winner.org/WINNER2-Deliverables/D1.1.2v1.1.pdf.

[48] J. Maurer, T. Fügen, and W. Wiesbeck, "Narrow-band measurement and analysis of the inter-vehicle transmission channel at $5.2 \mathrm{GHz}$," in Proceedings of the IEEE 55th Vehicular Technology
Conference (VTC '02), pp. 1274-1278, Birmingham, UK, May 2002.

[49] J. Maurer, T. M. Schäfer, and W. Wiesbeck, "A realistic description of the environment for inter-vehicle wave propagation modelling," in Proceedings of the IEEE 54th Vehicular Technology Conference (VTC '01), pp. 1437-1441, Atlantic, NJ, USA, October 2001.

[50] J. Maurer, T. Fügen, T. Schäfer, and W. Wiesbeck, "A new intervehicle communications (IVC) channel model," in Proceedings of the IEEE 60th Vehicular Technology Conference (VTC '04), pp. 9-13, Los Angeles, Calif, USA, September 2004.

[51] J. W. McKown and R. L. Hamilton Jr., "Ray tracing as a design tool for radio networks," IEEE Network, vol. 5, no. 6, pp. 27-30, 1991.

[52] A. Maltsev, R. Maslennikov, A. Lomayev, A. Sevastyanov, and A. Khoryaev, “IEEE 802. 11-09/0431r0”, April 2009.

[53] J. Nuckelt, M. Schack, and T. Kürner, "Deterministic and stochastic channel models implementedin a physical layer simulator for Car-to-X communications," Advances in Radio Science, vol. 9, pp. 165-171, 2011.

[54] K. Guan, Z. Zhong, B. Ai, and T. Kurner, "Deterministic propagation modeling for the realistic high-speed railway environment," in Proceedings of the IEEE 77th Vehicular Technology Conference (VTC '13), pp. 1-5, Dresden, Germany, 2013.

[55] W. C. Jakes, Ed., Microwave Mobile Communications, Wiley, New York, NY, USA, 1974.

[56] A. S. Akki and F. Haber, "A statistical model of mobile-to-mobile land communication channel," IEEE Transactions on Vehicular Technology, vol. 35, no. 1, pp. 2-7, 1986.

[57] A. S. Akki, "Statistical properties of mobile-to-mobile land communication channels," IEEE Transactions on Vehicular Technology, vol. 43, no. 4, pp. 826-831, 1994.

[58] F. Vatalaro, "Doppler spectrum in mobile-to-mobile communications in the presence of three-dimensional multipath scattering," IEEE Transactions on Vehicular Technology, vol. 46, no. 1, pp. 213-219, 1997.

[59] P. A. Bello, "Characterization of randomly time-variant linear channels," IEEE Transactions on Communications, vol. 11, pp. 360-393, 1963.

[60] G. Acosta and M. A. Ingram, "Model development for the wideband expressway vehicle-to-vehicle $2.4 \mathrm{GHz}$ channel," in Proceedings of the IEEE Wireless Communications and Networking Conference (WCNC '06), pp. 1283-1288, Las Vegas, Nev, USA, April 2006.

[61] G. Acosta-Marum and M. A. Ingram, "Six time- and frequencyselective empirical channel models for vehicular wireless LANs," IEEE Vehicular Technology Magazine, vol. 2, no. 4, pp. 4-11, 2007.

[62] C. Wang, X. Cheng, and D. Laurenson, "Vehicle-to-vehicle channel modeling and measurements: recent advances and future challenges," IEEE Communications Magazine, vol. 47, no. 11, pp. 96-103, 2009.

[63] A. Chelli and M. Pätzold, "A non-stationary MIMO vehicleto-vehicle channel model derived from the geometrical street model," in Proceedings of the IEEE 74th Vehicular Technology Conference (VTC '11), pp. 1-6, San Francisco, Calif, USA, September 2011.

[64] T. Kürner and M. Schack, "3D ray-tracing embedded into an integrated simulator for car-to-X communications," in Proceedings of the 20th URSI International Symposium on Electromagnetic Theory (EMTS '10), pp. 880-882, Berlin, Germany, August 2010. 

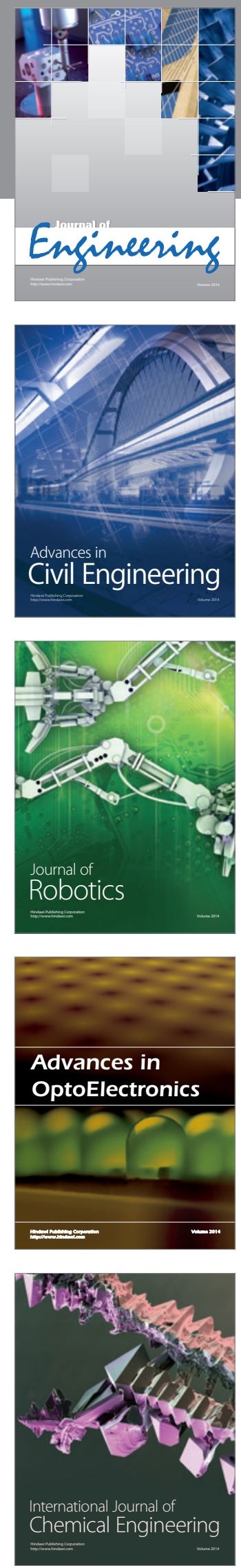

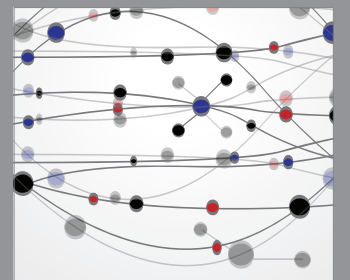

The Scientific World Journal
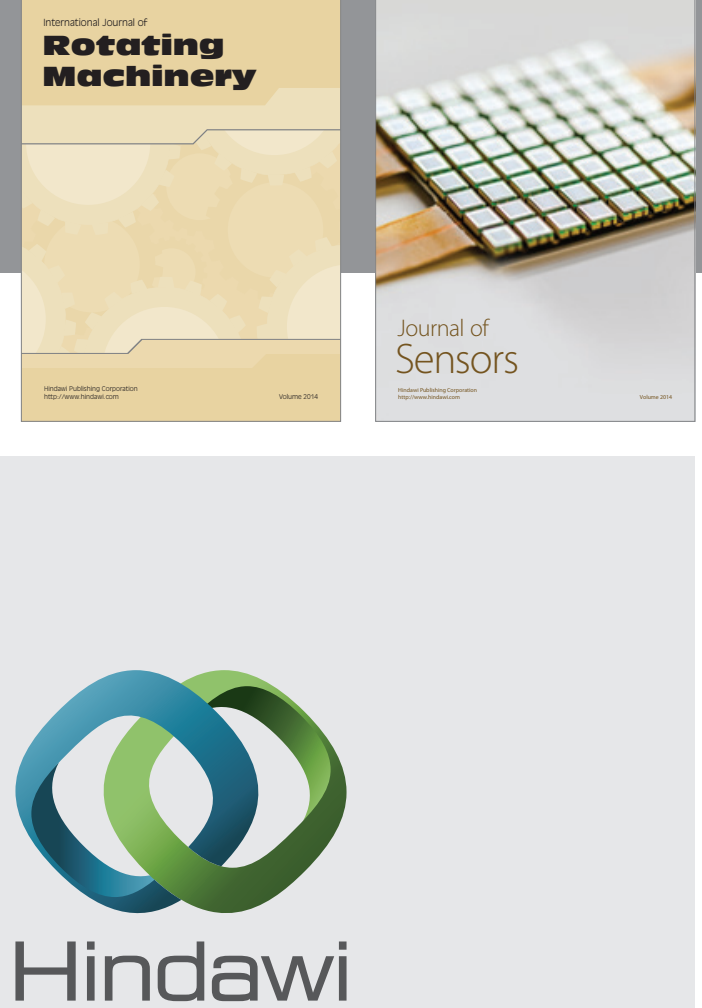

Submit your manuscripts at http://www.hindawi.com
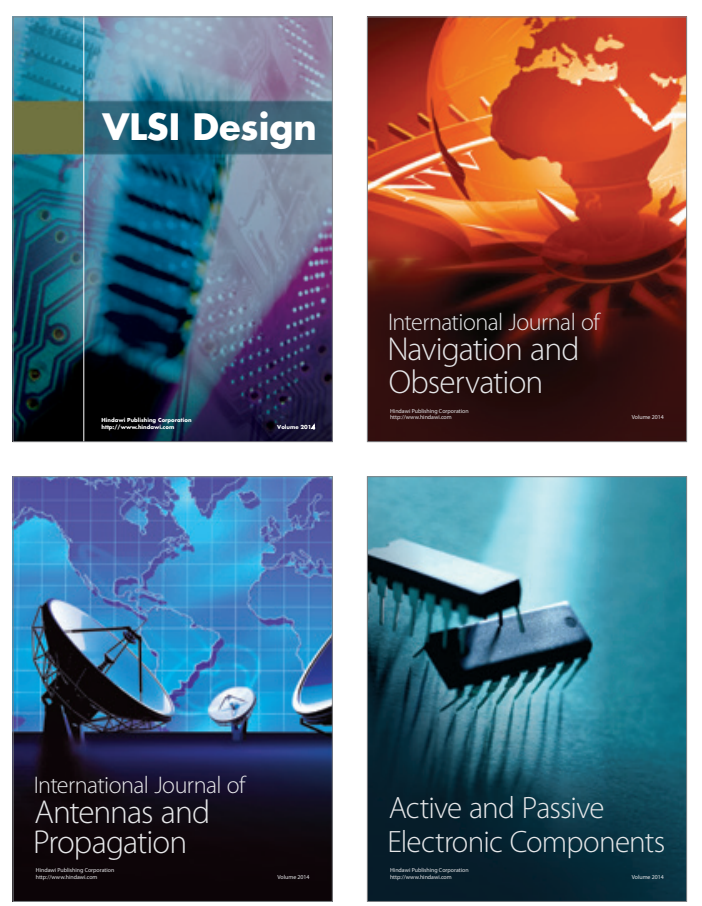
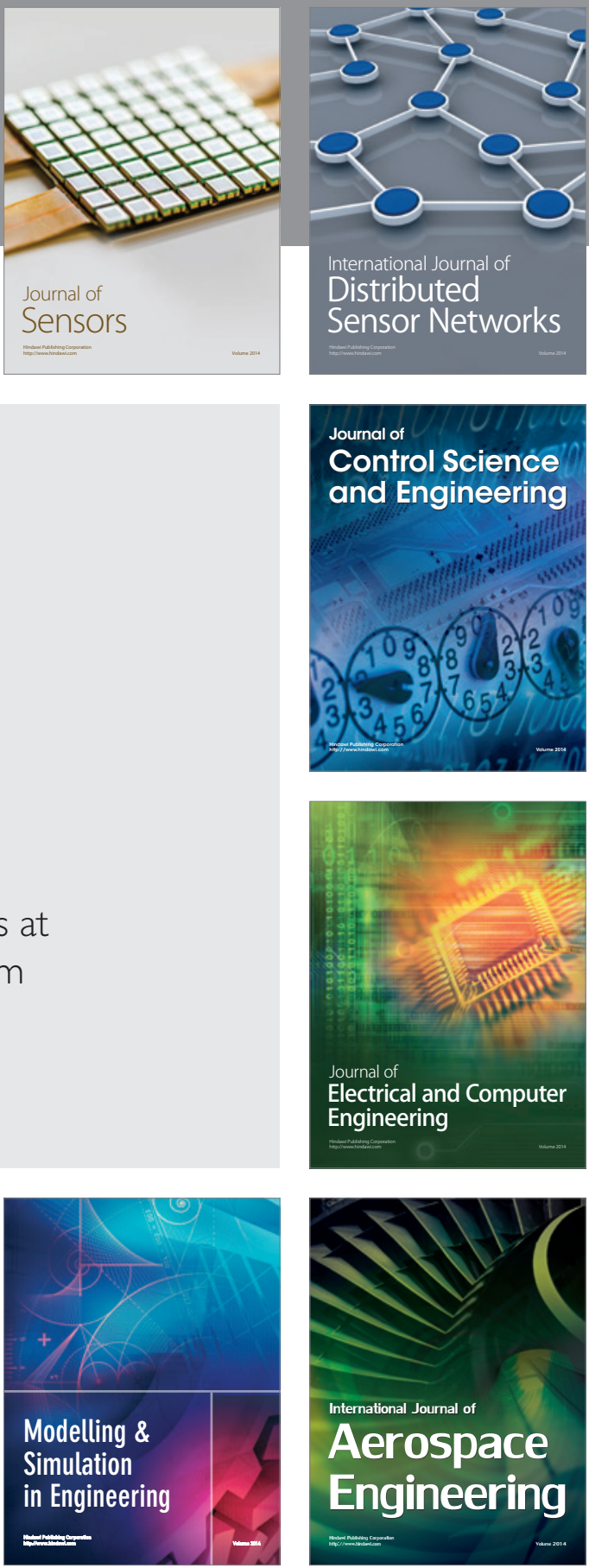

Journal of

Control Science

and Engineering
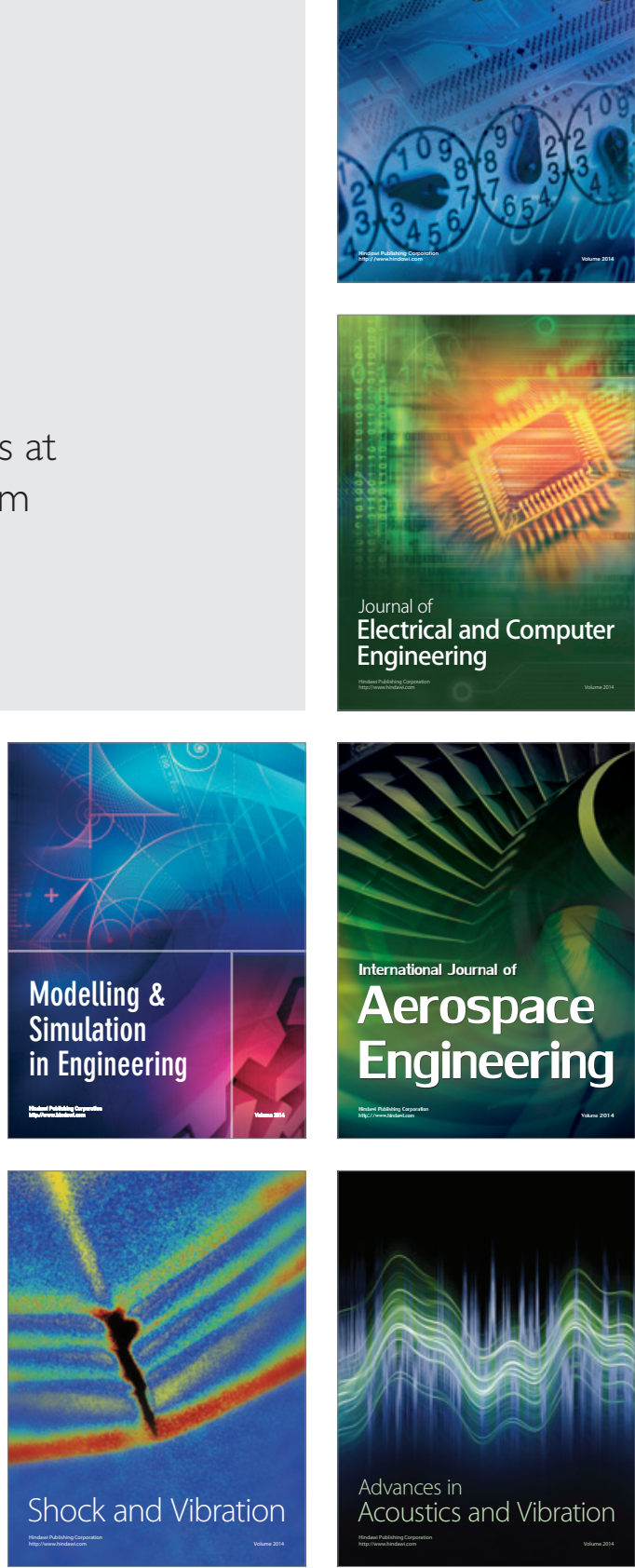